Cadaver Study

\title{
Successful Identification and Assessment of the Superior Cluneal Nerves with High-Resolution Sonography
}

Gerd Bodner, MD1, Hannes Platzgummer, MD1, Stefan Meng, MD², Peter C. Brugger, MD, $\mathrm{PhD}^{3}$, Gerlinde Maria Gruber, MD, MSc ${ }^{3}$, and Doris Lieba-Samal, $\mathrm{MD}^{4}$

From: ${ }^{1}$ Department of Applied Radiology and Image-guided Therapy, Medical University of Vienna; ${ }^{2}$ Department of Radiology, KFJ Hospital, Austria; ${ }^{3}$ Centre for Anatomy and Cell Biology, Department of Systematic Anatomy, Medical University of Vienna; ${ }^{4}$ Department of Neurology, Medical University of Vienna

Address Correspondence: Doris Lieba-Samal, MD Department of Neurology Medical University of Vienna

Waehringer Guertel 18-20 1090 Vienna

E-mail: doris.lieba-samal@ meduniwien.ac.at

Disclaimer: There was no external funding in the preparation of this manuscript. Conflict of interest: Each author certifies that he or she, or a member of his or her immediate

family, has no commercial association (i.e., consultancies, stock ownership, equity interest, patent/licensing arrangements, etc.) that might pose a conflict of interest in connection with the submitted manuscript.

Manuscript received: 08-24-2015 Revised manuscript received: 09-30-2015

Accepted for publication: 10-12-2015

Free full manuscript: www.painphysicianjournal.com
Background: Low back pain is a disabling and common condition, whose etiology often remains unknown. A suggested, however rarely considered, cause is neuropathy of the medial branch of the superior cluneal nerves $(\mathrm{mSCN})$ - either at the level of the originating roots or at the point where it crosses the iliac crest, where it is ensheathed by an osseo-ligamentous tunnel. Diagnosis and treatment have, to date, been restricted to clinical assessment and blind infiltration with local anesthetics.

Objective: To determine whether visualization and assessment of the $\mathrm{mSCN}$ with high-resolution ultrasound (HRUS) is feasible.

Study Design: Interventional cadaver study and case series.

Methods: Visualization of the mSCN was assessed in 7 anatomic specimens, and findings were confirmed by HRUS-guided ink marking of the nerve and consecutive dissection. Further, a patient chart and image review was performed of patients assessed at our department with the diagnosis of mSCN neuropathy.

Results: The mSCN could be visualized in 12 of 14 cases in anatomical specimens, as confirmed by dissection. Nine patients were diagnosed with $\mathrm{mSCN}$ syndrome of idiopathic or traumatic origin. Diagnosis was confirmed in all of them, with complete resolution of symptoms after HRUSguided selective nerve block.

Limitations: These findings are first results that need to be evaluated in a systematic, prospective and controlled manner.

Conclusion: We hereby confirm that it is possible to visualize the $\mathrm{mSCN}$ in the majority of anatomical specimens. The patients described may indicate a higher incidence of mSCN syndrome than has been recognized. mSCN syndrome should be considered in patients with low back pain of unknown origin, and HRUS may be able to facilitate nerve detection and US-guided nerve block.

Key words: Superior cluneal nerves, high-resolution ultrasound, peripheral neuropathy, entrapment syndrome, nerve trauma, low-back pain, diagnostic blockade, anatomic study, interventional study, Maigne syndrome

Pain Physician 2016; 19:197-202 ow-back pain (LBP) is a frequent and disabling condition, with an estimated lifetime prevalence of $84 \%$, and causes an enormous socioeconomic burden through direct and indirect costs (1). The etiology of LBP can be categorized into mechanical ( 80 $-90 \%)$, neurological $(5-15 \%)$, specific spinal $(1-2 \%)$, and non-spinal $(1-3 \%)$ causes (1). Among mechanical causes, $30-35 \%$ can be attributed to specific causes 
like e.g. degenerative disc and joint disease, vertebral fracture, instability or congenital deformity. However, in the vast majority of cases (65-70\%), no cause can be identified and these cases are classified as "unspecific LBP," and usually attributed to muscle strain or ligamentous injury (1). Considering the heavy individual burden and cost to society, it would be desirable to unravel the possible additional causes of LBP.

One cause-although presumed rare-for LBP is neuropathy of the medial branch of the superior cluneal nerves ( $\mathrm{mSCN}$ ). The superior cluneal nerves ( $\mathrm{SCN}$ ) consist variably of the lateral rami of dorsal roots T11 to L4, with T12 and L1 contributing to the majority of cases (2). These nerves also supply the skin of the lower back, the lateral inguinal area, the upper buttock, and, sometimes, the proximal lateral thigh as well $(2,3)$ (Fig. 1).

A probable idiopathic entrapment syndrome was postulated by Maigne et al (2) in 1989, who described the findings of 37 dissections, which showed the skin of the lower back to be innervated by the SCN in various combinations, with the most medial nerve invariably crossing the iliac crest within a rigid tunnel formed by the inferior edge of the thoracolumbar fascia and the superior margin of the iliac crest. In 2 cases, they found

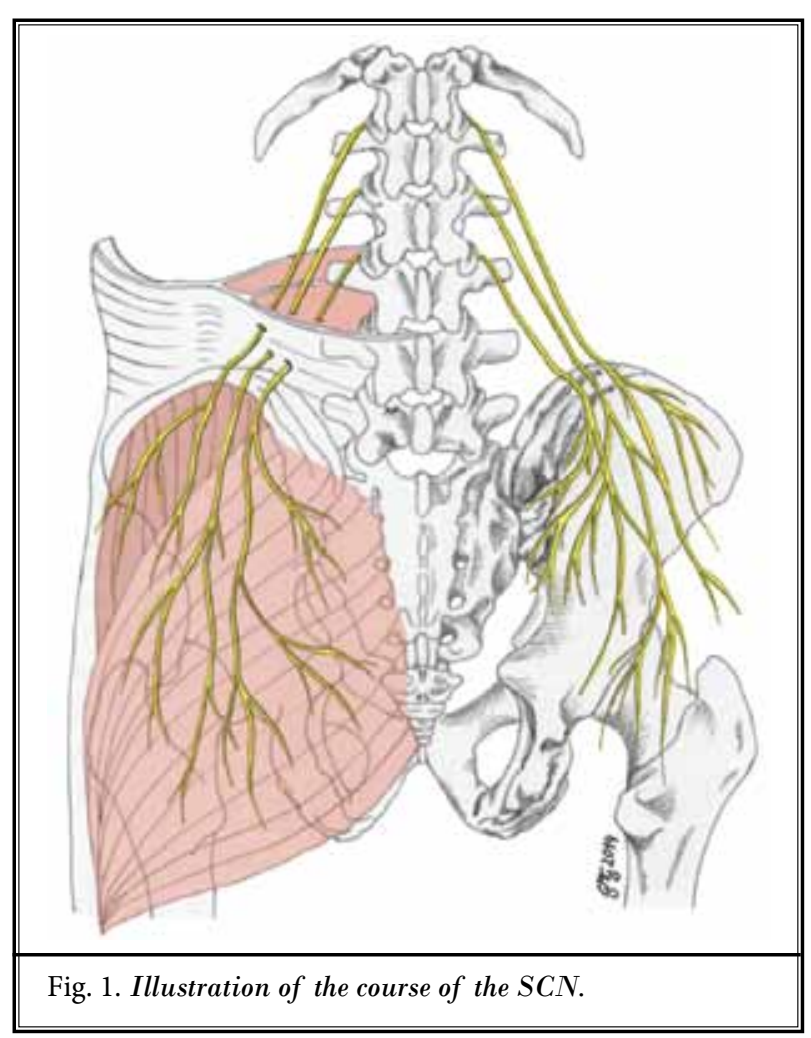

marked compression of the nerve within the orifice. They concluded that their findings would explain why lesions of the thoracolumbar joint can be felt as LBP and why compression at the level of the tunnel can produce pain radiating down the gluteal region and even to the upper thigh. Subsequent anatomical studies confirmed Maigne's findings (3-5).

To date, the diagnosis of $\mathrm{mSCN}$ entrapment has been performed only clinically. Kuniya (6) suggested diagnostic criteria that included determination of the maximal point of tenderness at the height of the presumed osteofibrous tunnel and palpation of the tender point that reproduced the chief complaint of LBP and/ or thigh symptoms. With regard to treatment, some reports exist in which single or repetitive injections of local anesthetics, in combination with corticosteroids, reportedly provided good results (7-9). In a prospective case series including 19 patients who underwent surgery for suspected mSCN entrapment, 13 had an excellent and 6 an unsatisfactory outcome (the latter group included 4 patients in whom no compression could be demonstrated during surgery) (10).

latrogenic lesioning of the SCN is a well-known problem in bone harvesting procedures at the posterior iliac crest $(11,12)$, and cases of iatrogenic nerve injury due to intragluteal injection have been reported $(13,14)$.

Spontaneous traumatic injury of the $\mathrm{mSCN}$ has, to our knowledge, not been reported in the literature thus far; however; the possibility exists, as in other superficial nerves.

To date, the accuracy of the clinical diagnosis of mSCN entrapment is unknown. Applying diagnostic blockade by relying on anatomic landmarks could lead, instead, to unspecific analgesic effects rather than to selective blocking of the $\mathrm{mSCN}$.

For both problems, direct imaging of the nerve could be helpful. One emerging and increasingly used modality is high-resolution ultrasound (HRUS), working with probes of at least $18 \mathrm{MHz}$, and enabling a spatial resolution between 250 and $500 \mu \mathrm{m}$. This allows the assessment of even small sensory nerves like the mSCN. As the mean diameter of the $\mathrm{mSCN}$ ranges from 2.1 to $0.8 \mathrm{~mm}$ (mean, $1.1 \mathrm{~mm}$ ) (15), which is about the size we described for small nerves in a previous paper (16), we hypothesized that visualization of the $\mathrm{mSCN}$ and assessment along its course should be possible.

The aim of this study was to clarify whether visualization and assessment of the $\mathrm{mSCN}$ is possible with HRUS. Further, we screened for patients with LBP that was considered to be caused by $\mathrm{mSCN}$ neuropathy. 


\section{Methods}

The presented study was approved by the Ethics Committee of the Medical University of Vienna (EC Nr. 1478/2014).

\section{Ultrasound Technique}

HRUS examinations were performed using a GE Logiq E9 ultrasound platform with high-frequency probes (GE ML 6-15-D, L 8-18i-D). An examiner experienced in peripheral nerve ultrasound for over 20 years (G.B.) carried out all the examinations. In all anatomical specimens and patients, the $\mathrm{mSCN}$ was assessed. Initial detection was performed at the point where the mSCN crosses over the posterior iliac crest. It was then followed cranially to its origin from the lower thoracic/ upper lumbar roots. The vertebral level was identified by using the last rib as a reference point for T12. UItrasound images obtained at the starting point and onwards cranially are shown in Fig. 2A-C.

\section{Ultrasound in Anatomical Specimens}

After receiving randomly selected anatomic specimens in the legal custody of the Department of Systematic Anatomy, Medical University of Vienna, HRUS was performed as described above. After presumed visualization of the $\mathrm{mSCN}$, a small amount of blue dye $(0.1 \mathrm{~mL})$ was injected adjacent to the nerve under HRUSguidance, just before it crosses the iliac crest, as this point would be proximal to the presumed site of entrapment in idiopathic cases. Anatomical dissection was performed to confirm the location of the dye injection.

\section{Ultrasound in Patients}

Patient charts between January 1, 2012, and December 1, 2014, were screened for referrals because of LBP of unknown origin, radiating to the upper part of the buttock. HRUS was performed according to the method described above. To confirm the origin of pain within the SCN territory, an HRUS-guided fine-needle selective block with $1 \mathrm{~mL}$ of lidocaine $2 \%$ was performed (transverse view, free-hand and in-plane technique). The block was rated successful if it led to hypesthesia within the presumed territory of the $\mathrm{mSCN}$, as assessed by sensitivity to light touch.

\section{Results}

\section{Ultrasound in Anatomical Specimens}

The mSCN was identified correctly in 12 of 14 sites in anatomical specimens. An example of a find- ing is depicted in Fig. 3. The mSCN usually appeared as a hyperechoic structure that had no fascicular pattern.

As a standardized procedure for identification, we recommend starting in the transverse view above the gluteus maximus muscle, and then moving the probe proximally, until the insertion of the gluteus medius muscle and the posterior edge of the iliac crest appeared-which was the point around which most medial branch would cross (Fig. 1). In addition to pure identification, it seems important to report about the cranial course of this nerve, which was quite characteristic to us. After entering the erector spinae, the nerve showed a long wavy shape, which we presumed to be necessary because of the range of motion of the spine (Fig. 2b). Within the course of the muscle, the nerve travelled in a fascial-type layer.

\section{Ultrasound in Patients}

A total of 9 patients were identified with $\mathrm{mSCN}$ syndrome. Details of patient characteristics are given in Table 1. Among these patients, 4 had suffered a local trauma in the upper lumbar region (Patients 1 and $4-6$ ) and consecutively developed pain of unknown origin in the low back/upper buttock; however, in their understanding, this pain was far away from the initial trauma. Two patients (Patient 3 and Patient 8) experienced an idiopathic onset of pain that was initially diagnosed as sacroiliitis and was refractive to conservative treatment and sacroiliac injection and/or facet joint block of the lower back. One patient developed severe and medically refractory gluteal pain 12 months after a posterior lumbar interbody fusion (PLIF) surgery (Patient 9) and 2 patients underwent lumbar surgery that was directly followed by pain.

Ultrasound findings were unremarkable in all but one patient (Patient 9). No ultrasound-morphologic correlate, such as a distinct swelling of the $\mathrm{mSCN}$ in idiopathic cases or traumatic neuroma in those with local trauma, could be observed. Patient 9 showed massive swelling of the medial superior cluneal nerve (Fig. 4).

All patients received a diagnostic block of the $\mathrm{mSCN}$ at the height of the posterior iliac crest, at the point before the $\mathrm{mSCN}$ entered the thoracolumbar fascia (Fig. 5). Consequently, all experienced numbness within the presumed $\mathrm{mSCN}$ territory and blockade; thus, these procedures were rated as successful. Together with numbness, patients were pain-free for one to 2.5 hours, which confirmed the diagnosis of $\mathrm{mSCN}$ neuropathy. 


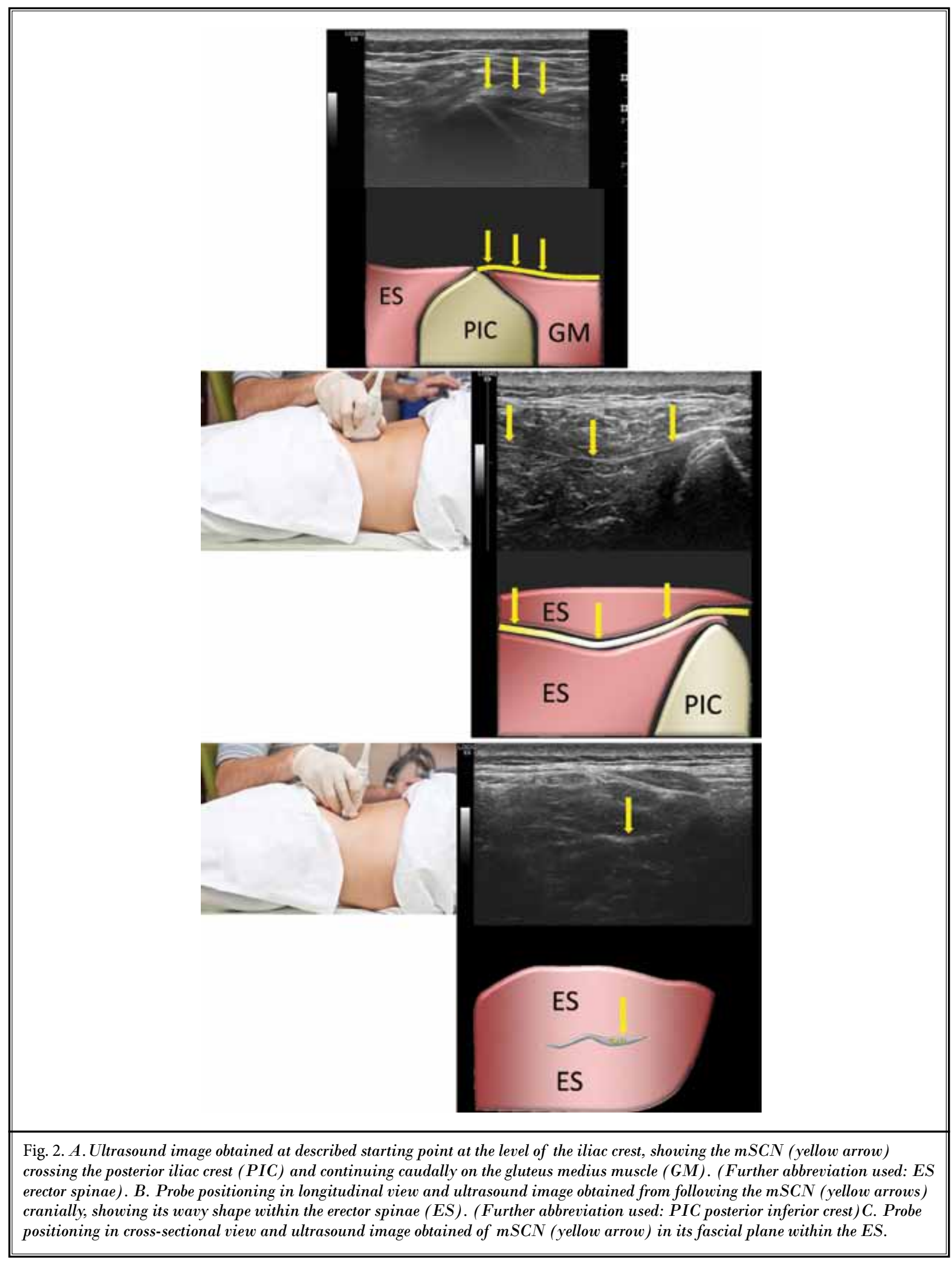




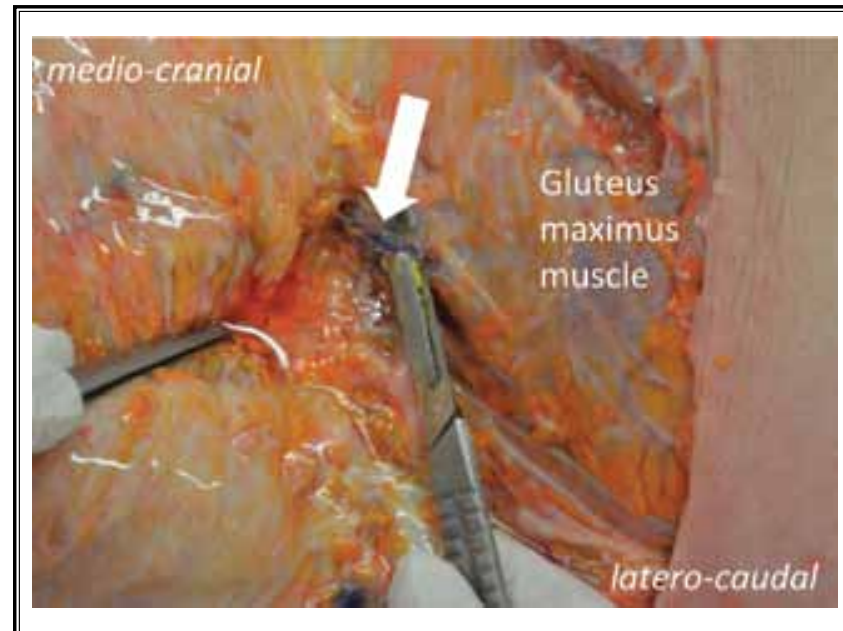

Fig. 3. Example of findings after ultrasound-guided marking and dissection, showing the medial branch of the SCN with ink marking (white arrow).

\section{Discussion}

This study confirms that the mSCN can be visualized and assessed with HRUS, although HRUS can be difficult or even impossible in obese patients or patients with extensive muscular atrophy, as the nerve cannot then be distinguished from the hyperechoic surrounding.

We believe that the most targeted method is to start with sagittal probe placement above the medial aspect of the insertion point of the gluteus maximus muscle, then move proximally to the insertion of the gluteus medius muscle where the $\mathrm{mSCN}$ is expected to cross over the iliac crest nearby. From there on, cranial assessment is possible.

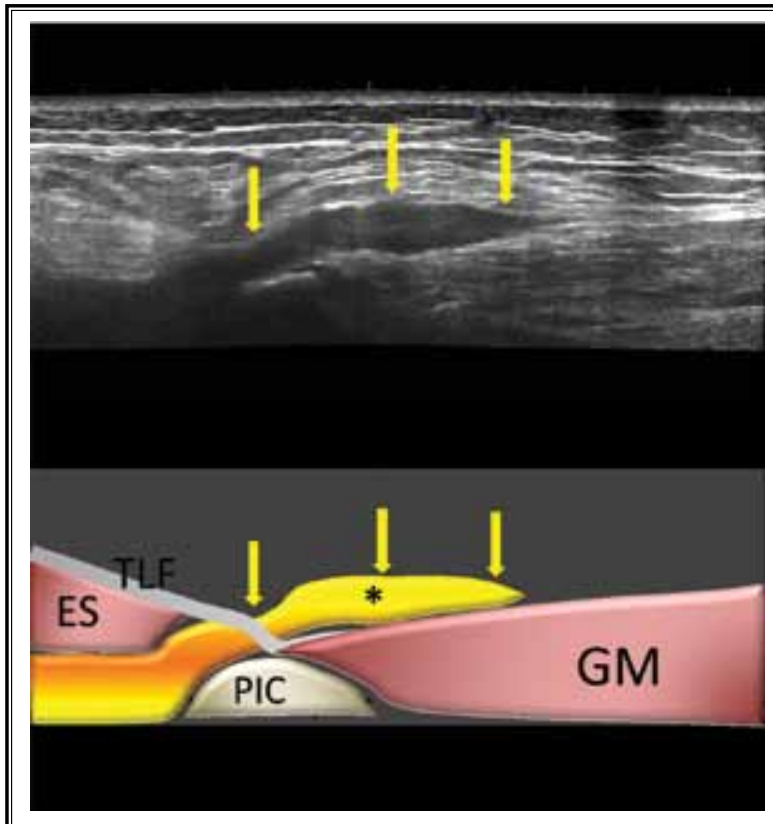

Fig.4. Longitudinal scan along the course of the mSCN (yellow arrows) showing a spindle-like hypoechoic lesion above the PIC when crossing the thoracolumbar fascia ( TLF), consistent with marked peripheral neuritis after PLIF surgery. (Abbreviations used: ES erector spinae, GM gluteus medius muscle)

After localization, diagnostic evaluation was considered the next step. However, except in one case, we did not encounter distinct swelling of the nerve on the symptomatic side compared to the asymptomaticwhich would surely be the most definitive sign that the nerve was affected, and is routinely used for entrapment syndromes and traumatic neuromas, primarily in

Table 1. Clinical characteristics in 9 patients with neuropathy of the superior cluneal nerve(s).

\begin{tabular}{|c|c|c|c|c||}
\hline No. & Gender & Age & Affected side & Clinical history \\
\hline 1 & F & 23 & Left & Fracture of L1 \\
\hline 2 & F & 72 & Right & Fusion surgery lumbar region \\
\hline 3 & M & 30 & Right & Idiopathic onset of lower lumbar and upper buttock pain, initial diagnosis of sacroilitis \\
\hline 4 & M & 15 & Right & $\begin{array}{c}\text { Was hit by a puck at height of L1 playing ice hockey. After some days he developed pain } \\
\text { far away from the initial point of trauma - in the upper buttock, radiating down to the } \\
\text { trochanteric area }\end{array}$ \\
\hline 5 & M & 55 & Right & Slipped and fell on a stone in the iliosacral area \\
\hline 6 & F & 23 & Left & $\begin{array}{c}\text { Suffered a hematoma with accompanying severe pain in the upper buttock area after falling } \\
\text { down stairs. After surgical removal of the hematoma, pain continued. }\end{array}$ \\
\hline 7 & M & 57 & Left & Repeated surgery of the lumbar region \\
\hline 8 & M & 71 & Right & $\begin{array}{c}\text { Suffered from chronic lumbar pain, diagnosed as sacroileitis or facette syndrome. Resistant } \\
\text { to conservative therapy }\end{array}$ \\
\hline 9 & F & 74 & Right & $\begin{array}{c}\text { Underwent lumbar fusion surgery and developed intractable pain in upper buttock area } \\
\text { one year afterwards }\end{array}$ \\
\hline \hline
\end{tabular}




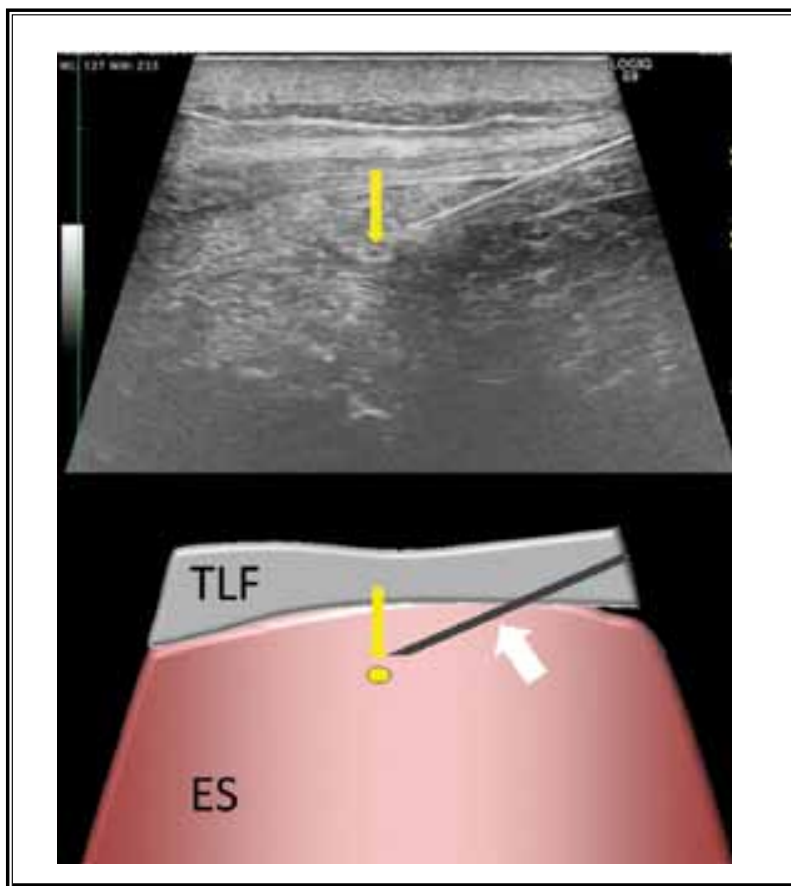

Fig. 5. Example of image obtained during HRUS-guided mSCN block: needle (white arrow) next to mSCN (yellow arrow). (Abbreviations used: ES erector spinae, TLF thoracolumbar fascia) larger nerves. Thus, as far as we can tell, this does not seem mandatory for these small nerves, at least with currently commercially available ultrasound probes.

In any case, diagnostic blockade is necessary to confirm the origin of pain within the territory of the $\mathrm{mSCN}$, which was true for all the patients in our cohort. Within our case series, we had only 3 patients with an idiopathic onset of pain that would correspond to the syndrome described as classical entrapment syndrome.

The other patients assessed were all referred for ultrasound assessment because of a presumed musculoskeletal origin of pain. Clinical assessment before ultrasound examination, however, led to the suspicion that the $\mathrm{mSCN}$ might be involved and indicated the need for further evaluation after excluding other causes.

Therefore, we think that 1) SCN neuropathy should be considered in patients with surgery or trauma in the upper lumbar region who develop pain in the lower back, or buttock, far away from the initial site of trauma; and 2) future systematic studies should be performed in patients with non-specific low-back pain or buttock pain, to evaluate probable SCN entrapment.

\section{References}

1. Morlion B. Chronic low back pain: Pharmacological, interventional and surgical strategies. Nature Reviews Neurology 2013; 9:462-473.

2. Maigne JY, Lazareth JP, Guerin Surville $\mathrm{H}$, Maigne $\mathrm{R}$. The lateral cutaneous branches of the dorsal rami of the thoraco-lumbar junction. An anatomical study on 37 dissections. SRA 1989; 11:289-293.

3. Lu J, Ebraheim NA, Huntoon M, Heck $\mathrm{BE}$, Yeasting RA. Anatomic considerations of superior cluneal nerve at posterior iliac crest region. Clinical Orthopaedics and Related Research 1998; 347:224-228.

4. Kuniya H, Aota $\mathrm{Y}$, Saito T, Kamiya Y, Funakoshi K, Terayama $\mathrm{H}$, Itoh $M$. Anatomical study of superior cluneal nerve entrapment. Journal of Neurosurgery Spine 2013; 19:76-80.

5. Maigne JY, Maigne R. Trigger point of the posterior iliac crest: Painful iliolumbar ligament insertion or cutaneous dorsal ramus pain? An anatomic study. Arch Phys Med Rehabil 1991; 72:734-737.

6. Kuniya $\mathrm{H}$. Low back pain patients with suspected entrapment of the superi- or cluneal nerve. J Spine Research 2011; 2:1032-1035.

7. Aly TA, Tanaka Y, Aizawa T, Ozawa H, Kokubun S. Medial superior cluneal nerve entrapment neuropathy in teenagers: A report of two cases. The Tohoku Journal of Experimental Medicine 2002; 197:229-231.

8. Berthelot JM, Delecrin J, Maugars Y, Caillon F, Prost A. A potentially underrecognized and treatable cause of chronic back pain: Entrapment neuropathy of the cluneal nerves. The Journal of Rheumatology 1996; 23:2179-2181.

9. Talu GK, Ozyalcin S, Talu U. Superior cluneal nerve entrapment. Reg Anesth Pain Med 2000; 25:648-650.

10. Maigne JY, Doursounian L. Entrapment neuropathy of the medial superior cluneal nerve. Nineteen cases surgically treated, with a minimum of 2 years' follow-up Spine 1997; 22:1156-1159.

11. Mahli A, Coskun D, Altun NS, Simsek A, Ocal E, Kostekci M. Alcohol neurolysis for persistent pain caused by superior cluneal nerves injury after iliac crest bone graft harvesting in orthopedic surgery:
Report of four cases and review of the literature. Spine 2002; 27: $\mathrm{E}_{478} 8-\mathrm{E}_{48} 8$.

12. Seiler JG, 3rd, Johnson J. Iliac crest autogenous bone grafting: Donor site complications. Journal of the Southern Orthopaedic Association 2000; 9:91-97.

13. McKain CW, Urban BJ. Pain and cluneal neuropathy following intragluteal injection. Anesth Analg 1978; 57:138-141.

14. Kolb LC, Gray SJ. Peripheral neuritis as a complication of penicillin therapy. JAMA 1946; 132:323-326.

15. Tubbs RS, Levin MR, Loukas M, Potts EA, Cohen-Gadol AA. Anatomy and landmarks for the superior and middle cluneal nerves: Application to posterior iliac crest harvest and entrapment syndromes. Journal of Neurosurgery Spine 2010; 13:356-359.

16. Lieba-Samal D, Morgenbesser J, Moritz T, Gruber GM, Bernathova M, Michaud J, Bodner G. Visualization of the long thoracic nerve using high-resolution sonography. Ultraschall in der Medizin 2015; 36:264-269. 